\title{
The influence of polydispersity and inhomogeneity on EXAFS of bimetallic catalysts
}

\author{
J. Moonen ${ }^{a, *}$, J. Slot ${ }^{a}$, L. Lefferts ${ }^{a}$, D. Bazin ${ }^{b}$, H. Dexpert ${ }^{b}$ \\ ${ }^{2} D S M$ Research, 6160 MD Geleen, Netherlands \\ 'LURE, Université de Paris-Sud, 91405 Orsay, France
}

\begin{abstract}
The effect of polydispersity and inhomogeneity of supported bimetallic catalysts on the EXAFS analysis is investigated with some simple model calculations. These show that EXAFS is very insensitive to polydispersity. Polydispersity and inhomogeneous distribution of the metals over the particles however have only limited influence on the ability to distinguish between core-shell particles and particles with random distribution of both metals.
\end{abstract}

\section{Introduction}

We used EXAFS to study supported bimetallic catalysts with the aim to determine the size of the metal particles and the distribution of the metal in the particles. The size of the metal particles was determined from the number of neighbours found from EXAFS and compared with data obtained from the X-ray diffraction and electron microscopy. The particle sizes as derived from EXAFS were much smaller than those found with line width analysis from the X-ray diffraction. In fact the sizes found with EXAFS indicated that no metal peaks should have been observed at all in the X-ray diffractograms. A possible explanation of the discrepancy was the polydisperse character of the catalyst.

To our surprise we found no literature at all that dealt with the influence of polydispersity on the analysis of EXAFS spectra. Therefore, we did some very simple calculations based only on the number of neighbours, being one of the prime parameters to be determined by EXAFS to find out how sensitive EXAFS is to polydispersity. Since practical bimetallic catalysts are not only

${ }^{*}$ Corresponding author. polydisperse but also are inhomogeneous with respect to the composition of the individual metal particles we also investigated the influence of inhomogeneity in order to find out if it was possible at all to say something about particle size and metal distribution of realistic bimetallic catalysts.

\section{Polydispersity}

In all calculations that will be discussed FCC lattice symmetry and cubotahedral particle shape are assumed. In Table 1 the number of neighbours in the first four coordination shells $\left(N_{1}-N_{4}\right)$ are given for a number of particle sizes. In the last row of the table the data for a bidispers system consisting of $32 \mathrm{wt} \%$ of particles of 13 atom and $68 \mathrm{wt} \%$ of particles of 1415 atoms are given. The numbers in this row are obtained by taking the weight average number of the relevant preceeding rows. From the numbers $N_{1}$ to $N_{4}$ of the second and the fourth row one can conclude that the monodisperse system and the bidisperse system are indistinguishable. Since the dispersity chosen here is large it can generally be stated that EXAFS is very insensitive to polydispersity. Large 
Table 1

Number of neighbours in first coordination shells as a function of particle size

\begin{tabular}{|c|c|c|c|c|c|}
\hline$N_{\text {atoms }}$ & $N_{1}$ & $N_{2}$ & $N_{3}$ & $N_{4}$ & Diameter $[\AA]$ \\
\hline 13 & 5.5 & 1.85 & 3.7 & 0.9 & 8.3 \\
\hline 147 & 8.9 & 4.0 & 13.0 & 6.1 & 13.8 \\
\hline 1415 & 10.5 & 5.0 & 18.5 & 9.15 & 24.9 \\
\hline Bidispers & 8.9 & 4.0 & 13.8 & 6.5 & $8.3+24.9$ \\
\hline
\end{tabular}

differences between particle size determined from the $\mathrm{X}$-ray diffraction and EXAFS may well occur.

\section{Inhomogeneity}

EXAFS can provide useful information on the distribution of the two metals $A$ and $B$ of a bimetallic catalyst AB. One can determine if one of the metals is preferentially in the core of the metal particle or if the metal atoms are distributed randomly.

In a real bimetallic catalyst the metal particles will not only be polydisperse but they will also be inhomogeneous as far as their composition is concerned. Here the influence of both homogeneity and dispersity on the ability to distinguish between random particles and core-shell particles is investigated. The results are given in Table 2. The homogeneous and monodisperse system contains 147 atom particles with 9.82 atom\% A atoms. The homogeneous, polydisperse systems consists of particles of the same composition and two different sizes, $59.3 \mathrm{wt} \%$ of particles of 55 atoms and $40.7 \mathrm{wt} \%$ of particles of 1415 atoms. The monodisperse, heterogeneous system consists of 147 atom particles; $50 \%$ of which contain 3.3 atom $\% \mathrm{~A}$ atoms and $50 \%$ of which contain 16.3 atom $\% A$ atoms. In the polydisperse, heterogeneous case both effects are combined.

The procedure to obtain the numbers in the table is explained for the 9.8 atom $\%$ A, 147 atom particles. An ensemble of 147 atoms of which 9.8 atom \% of type $A$ is taken. In the case of a random particle one atom is taken at random from this ensemble and placed in the centre of
Table 2

Number of neighbours in the first coordination shell for different bimetallic catalysts

\begin{tabular}{lllll}
\hline Catalyst & $N_{\text {AA }}$ & $N_{\text {AB }}$ & $N_{\text {BA }}$ & $N_{\text {BB }}$ \\
\hline Ia & 0.8 & 8.2 & 0.9 & 8.1 \\
Ib & 0.7 & 6.5 & 0.7 & 8.5 \\
Ic & 5.5 & 6.5 & 0.7 & 8.0 \\
IIa & 1.2 & 7.8 & 0.8 & 8.1 \\
IIb & 1.0 & 6.2 & 0.7 & 8.5 \\
IIc & 4.5 & 7.5 & 0.4 & 8.3 \\
IIIa & 0.7 & 9.0 & 0.8 & 8.2 \\
IIIb & 0.8 & 6.3 & 0.6 & 8.5 \\
IIIc & 3.5 & 8.5 & 0.3 & 8.6 \\
IVa & 1.2 & 7.7 & 0.8 & 8.1 \\
IVb & 1.2 & 5.9 & 0.6 & 8.5 \\
IVc & 4.0 & 8.0 & 0.3 & 8.5 \\
\hline
\end{tabular}

I: homogeneous, monodisperse. II: heterogeneous, monodisperse. III: homogeneous, polydisperse. IV: heterogeneous, polydisperse. $a$ : random $\mathrm{AB}$ distibution. $\mathrm{b}$ : $\mathrm{B}$ core. $\mathrm{c}$ : $\mathrm{A}$ core.

the particle. The second atom is taken at random from the remaining 146 and placed on a position in the second shell starting from the centre. This procedure is continued until all 147 atoms are placed. Then the number of contacts $N_{\mathrm{XY}}$ where $\mathrm{X}$ represents the absorbing atom and $Y$ represents the backscattering atom are counted. This procedure is repeated 5000 times, and the numbers $N_{\mathrm{XY}}$ are averaged. For core shell particles the particles are also filled from the inside, shell by shell. But in that case as many shells as can be filled completely are filled completely with the atoms that are in the core, then the random drawing procedure is used for the shell that consists of two types of atoms and subsequently the remaining shells are filled with the remaining atoms which are all from the second type. In the case of polydisperse and/or inhomogeneous particles the numbers obtained in this manner for the different particles are averaged in the appropriate way, thus resulting in the numbers given in Table 2 . These calculations show that for core-shell particles and random particles can still be distinguished for inhomogeneous, polydisperse systems. 